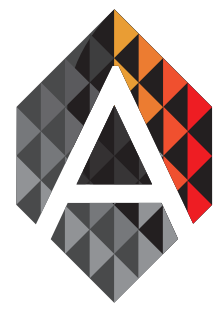

ADCAIJ: Advances in Distributed Computing and Artificial Intelligence Journal Regular Issue, Vol. 7 N. 1 (2018), 77-89

eISSN: $2255-2863$

DOI: http://dx.doi.org/10.14201/ADCAIJ2018717789

\title{
An extension of local mesh peak valley edge based feature descriptor for image retrieval in bio-medical images
}

\author{
Varun Srivastava, Ravindra Purwar \\ Guru Gobind singh Indraprastha university, New Delhi (India) \\ varun0621@gmail.com,ravindra@ipu.ac.in
}

\begin{tabular}{|c|c|}
\hline KEYWORD & ABSTRACT \\
\hline $\begin{array}{l}\text { Image retrieval; } \\
\text { Local Mesh } \\
\text { Peak Valley } \\
\text { Edge Pattern } \\
\text { (LMePVEP); } \\
\text { Local binary } \\
\text { pattern (LBP); } \\
\text { Directional local } \\
\text { ternary quantized } \\
\text { extrema pattern } \\
\text { (DLTerQEP) }\end{array}$ & $\begin{array}{l}\text { Various texture based approaches have been proposed for image indexing in bio-med- } \\
\text { ical image processing and a precise description of image for indexing in bio-medical } \\
\text { image database has always been a challenging task. In this paper, an extension of } \\
\text { local mesh peak valley edge pattern (LMePVEP) has been proposed and its effective- } \\
\text { ness is experimentally justified. The proposed algorithm explores the relationship of } \\
\text { center pixel with the surrounding ones along with the relationship of pixels amongst } \\
\text { each other in five different directions. It is then compared with the original LMePVEP } \\
\text { as well as a directional local ternary quantized extrema pattern (DLTerQEP) based } \\
\text { approach trema pattern using to bench mark databases viz. ELCAP database for lungs } \\
\text { and Wiki cancer data set for thyroid cancer. Further a live dataset for brain tumor is } \\
\text { also used for experimental evaluation. The experimental results show an improvement } \\
\text { up to } 19 \% \text { in terms of precision and retrieval for the proposed enhanced LMePVEP } \\
\text { technique over conventional LMePVEP approach in Wiki cancer data set. }\end{array}$ \\
\hline
\end{tabular}

\section{Introduction}

Bio-medical image indexing retrieves similar images from a bio-medical image dataset (Haralick 1973). This dataset comprises of Magnetic Resonance Imaging (MRI), Computed Tomography (CT)-Scan, Ultrasound and other images obtained from the radiology department of a hospital. The techniques are mainly based on spatial relationships used to extract features viz. gray-scale and color, texture, surfaces, regions etc. and find contrast between the images (Akgul 2011).

The basic approach for bio-medical indexing involves the use of local binary patterns (LBPs). It is a texture based approach which compares a given pixel with its neighbors to estimate the change in pixel intensity and thereby measure the texture of an image. Similar images are retrieved by comparing the distance between corresponding histograms (Ojala 1996). Knowledge driven approach like k-dimensional tree method have been used to build top-down decision tree for comparison of images in (Scott 2007). The texture based approach is extended by Tan et al. (Tan 2010) and Heikkiläin et al. (Heikkiläin 2009) who considered two threshold values and thus the concept of local ternary pattern (LTPs) was introduced. Performance of indexing based on LTP was found better as compared to LBPs and got widely accepted for image retrieval. Also Murala et al. (Murala 2012) extended it into a tetra pattern approach where a pixel is compared with all of its' horizontal and vertical

Varun Srivastava and Ravindra Purwar An extension of local mesh peak valley edge based feature descriptor for image retrieval in bio-medical images
ADCAIJ: Advances in Distributed Computing and Artifical Intelligence Journal Regular Issue, Vol. 7 N. 1 (2018), 77-89 elSSN: 2255-2863 - http://adcaij.usal.es Ediciones Universidad de Salamanca - CC BY NC DC 
neighbor. These neighbors are compared based on magnitude of their pixel intensity. A binary vector is thereby generated which acts as a feature vector. Later Guo et al. (Guo 2010) introduced the concept of rotation to compute LBP in directions like 45, 135 and 180 degrees, other than horizontal and vertical direction. Unay et al. (Unay 2008) introduced kanade-lucas-tomasi (KLT) points to enhance the feature vector as obtained by LBP. These KLT points indicate the dissimilarity between pixels. Thereby a common feature vector was formed by using both LBP and KLT points.

The concept of ternary patterns was also extended in many ways viz. local mesh patterns (LMeP) by Murala et al. (Murala 2014) or directional local ternary pattern (DLTP) histogram based feature vector by Vipparthi et al. (Vipparthi 2015). He followed the extraction of features for bio-medical image indexing with the application of Gabor filter to extract similar images. Local mesh peak valley edge pattern (LMePVEP) technique was introduced by Murala et al. in (Murala-2 2014). Vipparthi et al. in (Vipparthi 2016; Vipparthi-2 2015) proposed a method used primarily for face and texture retrieval by computing the magnitude of directional edges and accordingly constructing maximum edge patterns(MEPs) feature vector and maximum edge position patterns (MEPP).

Hussain et al. (Hussain 2012) introduced local quantized patterns (LQP) for face recognition where a look up table is generated to store different codes. One more extension to content based image retrieval (CBIR) is directional local extrema pattern (DLEP) which extracts the directional edge information based on local extrema in $0 \circ, 45^{\circ}, 90^{\circ}$, and $135^{\circ}$ directions in an image. These codes are generated based on the relationship between neighbors. Rao et al. (Rao 2015) combined the DLEP and LQP to obtain the local quantized extrema pattern (LQEP) for classification. Deep et al. in (Deep 2016) annexed an anti-diagonal descriptor to it for creating a better feature vector. Authors in (Kasturi 2002) presented a literature survey of various image/video retrieval algorithms.

Dimitrovski et al. (Dimitrovski 2015) and Antani et al. (Antani 2004) combined textual attributes of an image (like caption or tags associated with an image) with its spatial features (texture, color etc.) to form the feature vector. Convolution with Gabor filter and then binarization is used for classification and retrieval in (Zhang 2012). Gagandeep et al. (Deep-2 2016) introduced a directional local ternary quantized extrema pattern (DLTerQEP) and compared it with earlier approaches. This DLTerQEP approach compared intensity value of central pixel in a 7x7 image block to its neighbors in five different directions. Two binary vectors are thereby obtained using two threshold values which are then concatenated to form a final feature vector.

Semantic approaches for image indexing involves the use of different annotations which are attached to images after segmenting them. These annotations identify parts of an image and thus can be used to extract similar images from the database. These kind of semantic approaches are discussed and compared in Vogel 2007, Akakin 2012 and Quddus 2012.

Tumbull et al. (Gibbs 2003) worked for creating the feature vector from textual attributes. Do et al. (Do 2002) used wavelet transforms in different directions to obtain a frequency based feature vector. Various bio-medical databases have been used by the researchers like Wiki dataset (Kirk 2016 and Clark 2013), ELCAP database (Elcap 2017), ImageCLEF dataset (Kalpathy 2015 and Miller 2009) etc.. Manjunath et al. (Manjunath 2007) proposed statistical analysis for image retrieval and accordingly incorporated cumulative distribution function based algorithm for similarity index. Supervised learning algorithm like Support vector machines (SVMs) are also used as per (Rahman 2011) for image retrieval.

It can be seen in (Guo 2010), (Ojala 1996), (Unay 2008) etc. LBP has been extended in many ways to retrieve similar images from an image database. One such algorithm is (Murala 2012), but all these approaches considered only relationship between neighbors of a central pixel to form the feature vector. However it is evident from (Deep 2016) and (Deep-2 2016) that directional features play an important role to iden ify similar images, and thus can improve the retrieval rate and precision of the system. Therefore, authors in this paper proposed an image retrieval technique using LMePVEP based on directional features. It can be seen in the results and discussion section, that the proposed extension improves the average retrieval and precision rates as compared to LMePVEP.

Varun Srivastava and Ravindra Purwar An extension of local mesh peak valley edge based feature descriptor for image retrieval in bio-medical images
ADCAIJ: Advances in Distributed Computing and Artifical Intelligence Journal Regular Issue, Vol. 7 N. 1 (2018), 77-89 eISSN: 2255-2863 - http://adcaij.usal.es Ediciones Universidad de Salamanca - CC BY NC DC 


\section{Review of local pattern techniques}

\subsection{Local Binary Pattern (LBP)}

As suggested by authors in (Akgul 2011), LBP compares the gray scale value of central pixel with its P surrounding neighbors at radius $\mathrm{R}$ as shown in fig. 1 . If $\mathrm{I}\left(\mathrm{g}_{\mathrm{c}}\right)$ and $\mathrm{I}\left(\mathrm{g}_{\mathrm{i}}\right)$ where $1 \leq \mathrm{i} \leq \mathrm{P}$, represents intensity values of the central pixel and its $i^{\text {th }}$ neighbor respectively then, LBP $(P, R)$ is computed as in eq. (1).

$$
\begin{aligned}
& \operatorname{LBP}(\mathrm{P}, \mathrm{R})=\sum_{i=1}^{P} f\left(I\left(g_{c}\right)-I\left(g_{i}\right)\right) * 2^{i-1} \\
& f_{1}(x)=\left\{\begin{array}{l}
1 \text { if } x \geq 0 \\
0 \text { otherwise }
\end{array}\right.
\end{aligned}
$$

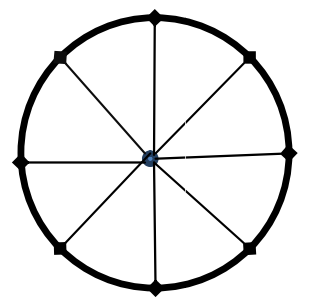

(a) $\operatorname{LBP}(8,1)$

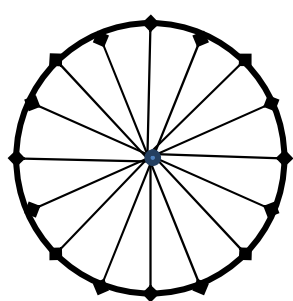

(b) $\operatorname{LBP}(16,2)$

Figure 1: Comparison of pixels from their neighbors in LBP with (a) $P=8, R=1$ (b) $P=16, R=2$.

\subsection{Local Mesh Peak Valley Edge Patterns (LMePVEP)}

Mesh patterns identify the relationship among neighboring pixels of a given center pixel (Murala 2012). As shown in fig. 2 , if the directions of texture variations of two horizontal neighboring pixels or two vertical neighboring pixels approach the center as shown in fig. 2(b) and fig. 2(f) respectively, we encode the relationship as a peak which is labeled as 2 and if they deviate from the center as shown in fig. 2(d) and fig. 2(h) we encode that as a valley which is labeled as 1 , otherwise label 0 is used as shown in fig. 2(a), fig.2(c), fig. 2(e) and fig. 2(g). Henceforth, peak valley encoding is done in the form of 0,1 and 2 values.

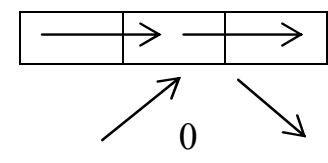

(a)

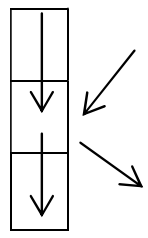

0

(e)

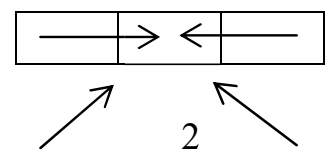

(b)

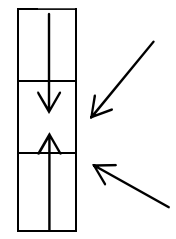

2

(f)

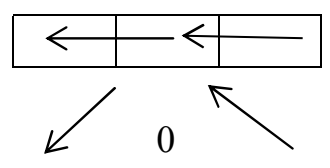

(c)

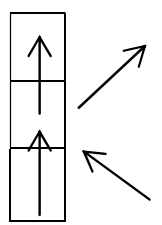

0

(g)

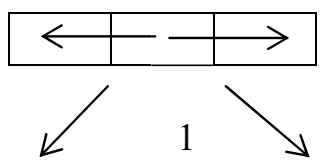

(d)

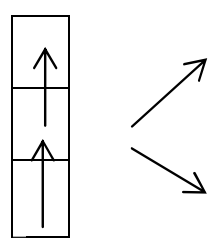

1

(h)

Figure 2: Peak valley encoding for different directions of texture variations.

Varun Srivastava and Ravindra Purwar An extension of local mesh peak valley edge based feature descriptor for image retrieval in bio-medical images
ADCAIJ: Advances in Distributed Computing and Artifical Intelligence Journal Regular Issue, Vol. 7 N. 1 (2018), 77-89 elSSN: 2255-2863 - http://adcaij.usal.es Ediciones Universidad de Salamanca - CC BY NC DC 
Local mesh peak valley edge pattern is computed by comparing the central pixel with P neighbors in radius $\mathrm{R}$ using first order gradient values in both forward and backward directions. If $\mathrm{I}_{\mathrm{P}, \mathrm{R}}^{\mathrm{f}}$ represents a first order forward derivative vector, then for central pixel $\mathrm{I}\left(\mathrm{g}_{\mathrm{c}}\right)$ it can be computed using eq. (3) in the following manner.

$$
\begin{aligned}
& I_{P, R}^{f}\left(g_{c}, g_{i}\right)=I(g(\alpha))-I(g(i)) \quad i=1,2, \ldots . P \\
& \text { where } \alpha=1+\bmod ((i+P+j-1), P)
\end{aligned}
$$

and $\mathrm{j}=1,2 \ldots \mathrm{P} / 2$, which represents the distance for first order derivation

Similarly, first order backward derivative $I^{b} P, R$, can be defined as-

$$
I_{P, R}^{b}\left(g_{c}, g_{i}\right)=\left\{\begin{array}{l}
I(g(P+i-j))-I g(i) \text { if } j \geq i \\
I(g(i-j))-I g(i) \text { otherwise }
\end{array}\right.
$$

where values of $i$ and $j$ vary as in eq. (3) and eq. (4) respectively.

Finally, local mesh peak valley edge pattern $\operatorname{LMePVEP}(\mathrm{P}, \mathrm{R})$ is represented as a column vector of size $\mathrm{P}$ as in eq. (6).

$$
\operatorname{LMePVEP}_{(P, R)}=\left[\begin{array}{cc}
f_{2}\left(f_{P, R}^{f}\left(g_{c}, g_{1}\right),\right. & \left.I_{P, R}^{b}\left(g_{c}, g_{1}\right)\right), \\
f_{2}\left(f_{P, R}^{f}\left(g_{c}, g_{2}\right),\right. & \left.I_{P, R}^{b}\left(g_{c}, g_{2}\right)\right), \\
\ldots \ldots \ldots \ldots \ldots \ldots \ldots \ldots \ldots \ldots \ldots \ldots \ldots \ldots & \ldots \ldots \ldots \ldots \ldots \\
f_{2}\left(\left(_{P, R}^{f}\left(g_{c} g_{P}\right)\right),\right. & \left.I_{P, R}^{b}\left(g_{c}, g_{P}\right)\right) \\
1 \times \mathrm{P}
\end{array}\right]
$$

where the function $\mathrm{f}_{2}(\mathrm{x}, \mathrm{y})$ used in eq. (6) is defined as:

$$
f_{2}(x, y)=\left\{\begin{array}{rr}
1 & \text { if } x>0 \text { and } y>0 \\
2 & \text { if } x<1 \text { and } y<1 \\
0 & \text { otherwise }
\end{array}\right.
$$

Therefore the possible values of all components of LMePVEP vector can be 0,1 or 2 . Fig. 3 represents an illustration for computation of LMePVEP values. The illustration considers a $3 \times 3$ block of original image and the LMePVEP values for 8 neighbors at radius $\mathrm{R}$ as 1 are computed thereby.

Varun Srivastava and Ravindra Purwar An extension of local mesh peak valley edge based feature descriptor for image retrieval in bio-medical images
ADCAIJ: Advances in Distributed Computing and Artifical Intelligence Journal Regular Issue, Vol. 7 N. 1 (2018), 77-89 elSSN: 2255-2863 - http://adcaij.usal.es Ediciones Universidad de Salamanca - CC BY NC DC 


\begin{tabular}{|l|l|l|}
\hline 7 & 2 & 4 \\
\hline 9 & 6 & 8 \\
\hline 1 & 4 & 2 \\
\hline
\end{tabular}

(a)

\begin{tabular}{|c|c|c|}
\hline 2 & 5 & -2 \\
\hline-8 & 6 & -4 \\
\hline 3 & -2 & 6 \\
\hline
\end{tabular}

(b)

\begin{tabular}{|c|c|c|}
\hline-5 & 2 & -4 \\
\hline-2 & 6 & -6 \\
\hline 8 & -3 & 2 \\
\hline
\end{tabular}

(d)

\begin{tabular}{|l|l|l|}
\hline 0 & 1 & 2 \\
\hline 2 & 6 & 2 \\
\hline 1 & 2 & 1 \\
\hline
\end{tabular}

(f)

\begin{tabular}{|c|c|c|}
\hline-6 & 7 & 3 \\
\hline-5 & 6 & -6 \\
\hline 1 & 4 & 2 \\
\hline
\end{tabular}

(c)

\begin{tabular}{|r|r|r|}
\hline-3 & 6 & -2 \\
\hline-7 & 6 & -4 \\
\hline 6 & 5 & -1 \\
\hline
\end{tabular}

(e)

\begin{tabular}{|l|l|l|}
\hline 2 & 1 & 0 \\
\hline 2 & 6 & 2 \\
\hline 1 & 1 & 0 \\
\hline
\end{tabular}

(g)

Figure 3: Calculation of LMePVEP patterns (a) Original $3 \times 3$ image block. (b) Forward first order derivative of the original image with $j=1$ (c) Forward first order derivative of the original image with $j=2$ (d) Backward first order derivative of the original image with $j=1$ (e) Backward first order derivative of the original image with $j=2(f)$ LMePVEP values for $j=1$ ( $g$ ) LMePVEP values for $j=2$.

A $3 \times 3$ image block is considered for the extraction of LMePVEP values as shown in fig.

3(a). Forward first order derivative values for $\mathrm{j}=1$ and $\mathrm{j}=2$ are computed for the central pixel using eq. (3) and eq. (4) and these derivative values are shown in fig. 3(b) and fig. 3(c) respectively. Similarly the backward first order derivative using for $\mathrm{j}=1$ and $\mathrm{j}=2$ are computed using eq. (5) and shown in fig. 3(d) and fig. 3(e) respectively. Finally LMePVEP coefficients are computed using eq. (7) which are shown in fig. 3(f) and fig. 3(g) for $\mathrm{j}=1$ and $\mathrm{j}=2$ respectively.

The peak information represented by 2 and the valley information represented by 1, from matrices in fig. 3(f) and fig. 3(g) is now separated. Fig. 4(a) represents a matrix in which pixels having only valley information are considered from the matrix of fig. 3(f), such pixels are assigned a value 1 and rest pixels are assigned a value 0 . Similarly fig. 4(b) represents a matrix obtained from matrix in fig. $3(\mathrm{~g})$ by making the pixels having valley information as 1 and rest as 0 . Further pixels having values 2 in fig. 3(f) and fig. 3(g) are represented as 1 in fig. 4(c) and fig. 4(d) for $\mathrm{j}=1$ and $\mathrm{j}=2$ respectively.

A weight matrix $\mathrm{W}$ consisting of $\mathrm{P}$ values (in this case 8 ) is generated as shown in fig. 4(e) using eq. (8) and an inner dot product of Weight matrix and LMePVEP values obtained in fig. 4(a) to fig. 4(d) is calculated and sum of coefficients of individual results is used to replace the central shaded pixel. Thus four values are obtained for central pixel as shown in fig. 4(f) to fig. 4(i). This process is repeated for the entire image and

Varun Srivastava and Ravindra Purwar An extension of local mesh peak valley edge based feature descriptor for image retrieval in bio-medical images
ADCAIJ: Advances in Distributed Computing and Artifical Intelligence Journal Regular Issue, Vol. 7 N. 1 (2018), 77-89 elSSN: 2255-2863 - http://adcaij.usal.es Ediciones Universidad de Salamanca - CC BY NC DC 
finally four images based on LMePVEP values are obtained. Then the histograms of these four images are formed. The four histograms are then concatenated to form a feature vector which is used for image retrieval. . To concatenate histograms pixels having same gray level values are summed and in this way a feature vector of length equal to number of different gray level values is obtained. The four histograms are then concatenated to form a feature descriptor which is used for image retrieval. This feature descriptor is thus of length $4 \times 256$.

$$
W(i)=1.2^{i-1} \quad \text { where } i=1 \ldots P
$$

\begin{tabular}{|l|l|l|}
\hline 0 & 1 & 0 \\
\hline 0 & 6 & 0 \\
\hline 1 & 0 & 1 \\
\hline
\end{tabular}

(a)

\begin{tabular}{|l|l|l|}
\hline 0 & 0 & 1 \\
\hline 1 & 6 & 1 \\
\hline 0 & 1 & 0 \\
\hline
\end{tabular}

(c)

\begin{tabular}{|l|l|l|}
\hline 0 & 1 & 0 \\
\hline 0 & 6 & 0 \\
\hline 1 & 1 & 0 \\
\hline
\end{tabular}

(b)

\begin{tabular}{|l|l|l|}
\hline 1 & 0 & 0 \\
\hline 1 & 6 & 1 \\
\hline 0 & 0 & 0 \\
\hline
\end{tabular}

(d)

\begin{tabular}{|l|l|l|}
\hline 8 & 4 & 2 \\
\hline 16 & & 1 \\
\hline 32 & 64 & 128 \\
\hline
\end{tabular}

(e)

\begin{tabular}{|l|l|l|}
\hline & & \\
\hline & 164 & \\
\hline & & \\
\hline
\end{tabular}

(f)

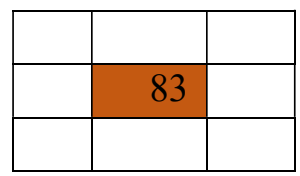

$(\mathrm{g})$

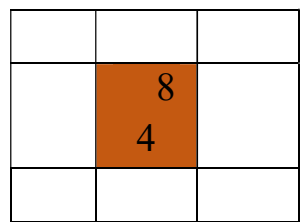

(h)

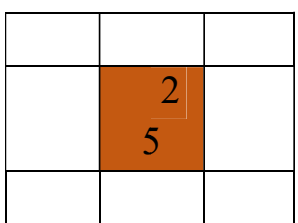

(i)

Figure 4: (a) Local mesh valley pattern extracted from original image for $j=1$ (b) Local mesh valley pattern for $j=2$ (c) Local mesh peak for $j=1$ (d) Local mesh peak pattern for $j=2$ (e) Weight Matrix (f) Weighted LMePVEP value of central pixel for $j=1$ in forward direction $(g)$ Weighted LMePVEP value of central pixel for $j=1$ in backward direction (h) Weighted LMePVEP value of central pixel for $j=2$ in forward direction (i)

Weighted LMePVEP value of central pixel for $j=2$ in backward direction.

\section{Proposed image indexing method}

The proposed method is based on an extension of LMePVEP which has been named in this paper as enhanced LMePVEP technique and it is summarized in the following sections.

Varun Srivastava and Ravindra Purwar An extension of local mesh peak valley edge based feature descriptor for image retrieval in bio-medical images
ADCAIJ: Advances in Distributed Computing and Artifical Intelligence Journal Regular Issue, Vol. 7 N. 1 (2018), 77-89 eISSN: 2255-2863 - http://adcaij.usal.es Ediciones Universidad de Salamanca - CC BY NC DC 


\subsection{Enhanced LMePVEP based feature value:}

In conventional LMePVEP computation, the values of peak and valley are computed using texture directions of horizontally and vertically adjacent pixels as shown in fig. 2 but in the proposed method, these values are added to texture gradient values computed in five different orientations- $0,45,90,135$ and 180 degrees as shown in Fig. 5.

Fig. 5(a) represents a $3 \times 3$ image block of input image. The enhanced LMePVEPs are computed in 0, 45, 90, 135 and 180 degrees using pixel locations shown in fig. 5(b) to fig. 5(f). In each case, the pixel intensity lying on the tail of the directional arrow is replaced by the difference of pixel intensity lying on the head and on the tail of the directional arrow, and these difference blocks are shown in fig. $5(\mathrm{~g})$ to fig. $5(\mathrm{k})$ for $0,45,90,135$ and 180 degrees respectively. Thereafter, a directional gradient vector of length 5 is generated where $1^{\text {st }}$ component represents the sum of elements of the difference block for 0 degree as shown in fig. $5(\mathrm{~g}), 2^{\text {nd }}$ component represents the sum of elements of the difference block for 45 degree as shown in fig. 5(h) and so on.

Each component of this vector is then compared with a predefined threshold $\mathrm{T}$ whose value is 10 in this paper and it is replaced with 0 if the component value is less than threshold $\mathrm{T}$ otherwise it is replaced by 1 as shown in fig. 5(1).

A weight vector of length 5 is generated using eq. (8) and inner dot product of this weight vector with the directional gradient vector shown in fig. 5(1) is computed as shown in Fig. 5(m) which represents the weighted directional gradient vector $\mathrm{H}$ of original $3 \times 3$ image block. Finally all components of $\mathrm{H}$ are added and this summation is denoted by S. The sum $\mathrm{S}$ is added with one LMePVEP value as shown in Fig. 4(f) to Fig. 4(i) at a time and replaces the central pixel value of $3 \times 3$ block with the result. This process is continued for all overlapping $3 \times 3$ image blocks which are one pixel distant from each other in horizontal and vertical directions. Ultimately four images using enhanced LMePVEP values are obtained from original image and their histograms are computed. These four histograms are concatenated to obtain the feature vector of the original image. The concatenation is done using same method as that of conventional LMePVEP. These histograms are computed keeping 256 equally spaced bins. Thus one histogram is of length 256 . These four histograms are concatenated to obtain the feature vector of the original image. Thus the length of feature vector $\mathrm{f}_{\mathrm{q}}$ is $4 \times 256$. The conca-tenation is done using same method as that of conventional LMePVEP. Moreover, the length of feature vector of various algorithms used for experimental results in this paper is shown in Table 1. It can be seen that number of features used in the proposed methodare same as conventional LMePVEP and substantially less than DLTerQEP.

Table 1: Feature vector length for different algorithms.

\begin{tabular}{c|c|c}
\hline S. No. & Algorithm & Feature Vector Length \\
\hline 1. & LBP & 256 \\
\hline 2. & LTP & $2 \times 256$ \\
\hline 3. & LMePVEP & $4 \times 256$ \\
\hline 4. & DLTerQEP & $2 \times 4096$ \\
\hline 5. & Proposed extension of LMePVEP & $4 \times 256$ \\
\hline
\end{tabular}

\subsection{Image Tetrieval}

The process of bio-medical image retrieval is summarized in the following steps:

1. Compute enhanced LMePVEP feature vector $\mathrm{f}_{\mathrm{i}}(1 \leq \mathrm{i} \leq \mathrm{n})$ for all ' $\mathrm{n}$ ' bio-medical images in image database and represent each image in terms of four images as discussed in previous section. The histogram is computed for these four images and thereby the four histograms are concatenated to obtain feature vector for original image which is then stored in a file. This process is repeated for all $\mathrm{n}$ images in database.

Varun Srivastava and Ravindra Purwar An extension of local mesh peak valley edge based feature descriptor for image retrieval in bio-medical images
ADCAIJ: Advances in Distributed Computing and Artifical Intelligence Journal Regular Issue, Vol. 7 N. 1 (2018), 77-89 elSSN: 2255-2863 - http://adcaij.usal.es Ediciones Universidad de Salamanca - CC BY NC DC 
2. Compute enhanced feature vector $\mathrm{f}_{\mathrm{q}}$ for query image.

3. Find Euclidean distance D (q, i) between feature vector $f_{q}$ of the query image and feature vector $f_{i}(1 \leq i$ $\leq \mathrm{n})$ of database image as per eq. (12).

$$
\mathrm{D}(\mathrm{q}, \mathrm{i})=\sum_{k=1}^{L}\left|\mathrm{f}_{\mathrm{ik}}-\mathrm{f}_{\mathrm{qk}}\right|
$$

where $\mathrm{L}$ is the length of feature vector.

4. Retrieve the images with minimum Euclidean distance.

\subsection{Description of databases used.}

Experimental analysis of the image retrieval system using enhanced LMePVEP feature vector is performed over three datasets and its performance is compared with LBP (Ojala 1996), conventional LMePVEP based indexing method (Murala 2012) and DLTerQEP (Deep 2016) based method. All simulation work has been carried out over a computational device with 4 GB RAM, $2 \mathrm{GHz}$ processor and MATLAB version 2016b using Windows environment. These databases that have been used are:

1. MRI image dataset for different stages of brain tumor: This is a live data which has been collected data from Deen Dayal Upadhyay Hospital, New Delhi for different patients suffering from brain tumor. The data set has MR images for different stages of cancer.

2. ELCAP Lung CT data set ( Elcap 2017): It consists of CT-Scan images for lungs of different patients. These patients had nodules in their lungs at different positions.

3. Wiki Cancer Imaging Archive (TCIA) public database (Kirk 2016 and Clark 2013): The images belong to patients suffering from thoracic cancer. The MR images of 6 patients have considered together to verify retrieval capabilities of the proposed algorithm. The neck soft tissue MR images of different patients are considered. The ELCAP lung database has been widely used by the researchers before in similar approaches where relationship between neighboring pixels was identified to extract similar images. Also Wiki Cancer Imaging Archive is a popular open database to study various cancer related issues and perform retrieval based tasks. Further authors are willing to test the performance of their proposed retrieval technique over a live dataset and thus dataset 1 is considered which is taken from radiology department of Deen Dayal Upadhyay Hospital, New Delhi with due permission.

These three databases are used to justify that given extension of LMePVEP performs better than most of the similar algorithms proposed in the past since it incorporated directional features into regular LMEPVEP feature vector.Finally, different features of these input datasets are summarized in Table 2.

Table 2: Various aspects of the data sets obtained from different sources.

\begin{tabular}{c|c|c|c|c|c|c}
\hline $\begin{array}{c}\text { Dataset } \\
\text { No. }\end{array}$ & Dataset Source & $\begin{array}{c}\text { No. of } \\
\text { Slices }\end{array}$ & Length & Thickness & Voltage & Resolution \\
\hline 1 & $\begin{array}{c}\text { Deen Dayal Upadhyay Hospital, } \\
\text { New Delhi, India. }\end{array}$ & 100 & $65.7 \mathrm{~mm}$ & $5.5 \mathrm{~mm}$ & $120 \mathrm{kV}$ & $512 \times 512$ \\
\hline 2 & $\begin{array}{c}\text { ELCAP Lung CT } \\
\text { data set }\end{array}$ & 162 & $2.4 \mathrm{~mm}$ & $1.3 \mathrm{~mm}$ & $120 \mathrm{kV}$ & $512 \times 512$ \\
\hline 3 & $\begin{array}{c}\text { Wiki Cancer Imaging archive } \\
\text { (TCIA) public database }\end{array}$ & 100 & $60.5 \mathrm{~mm}$ & $2.5 \mathrm{~mm}$ & $120 \mathrm{kV}$ & $512 \times 512$ \\
\hline
\end{tabular}

Varun Srivastava and Ravindra Purwar An extension of local mesh peak valley edge based feature descriptor for image retrieval in bio-medical images
ADCAIJ: Advances in Distributed Computing and Artifical Intelligence Journal Regular Issue, Vol. 7 N. 1 (2018), 77-89 eISSN: 2255-2863 - http://adcaij.usal.es Ediciones Universidad de Salamanca - CC BY NC DC 


\section{Experimental Analysis}

Fig. 6(a) shows an input image from dataset 1 and fig. 6(b) to fig. 6(f) show the images which have been obtained by using directional gradient vector as discussed in subsection 3.1 along $0,45,90,135$ and 180 degrees. Fig. 6(g) to fig. 6(j) represent images using enhanced LMePVEP method in forward and backward direction for $\mathrm{j}=1$ and $\mathrm{j}=2$ respectively.

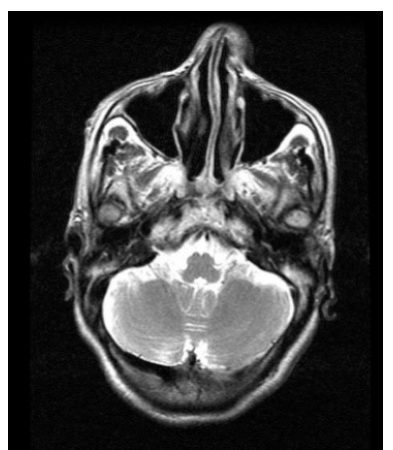

(a)

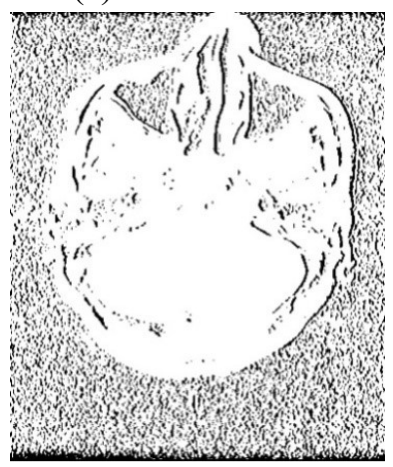

(d)

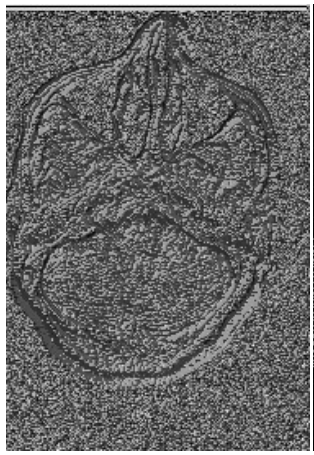

$(\mathrm{g})$

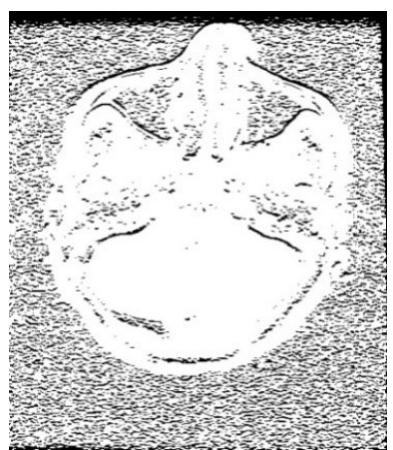

(b)

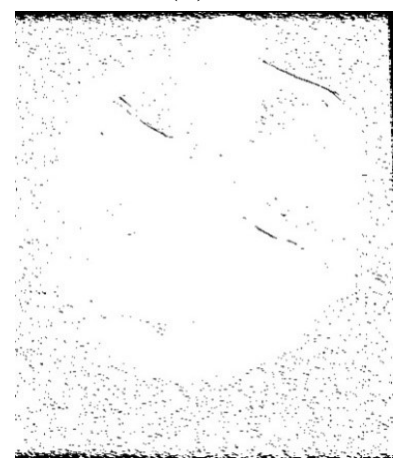

(e)

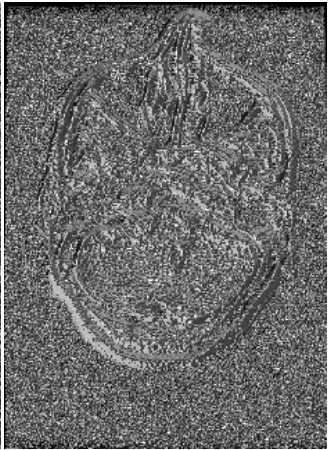

(h)

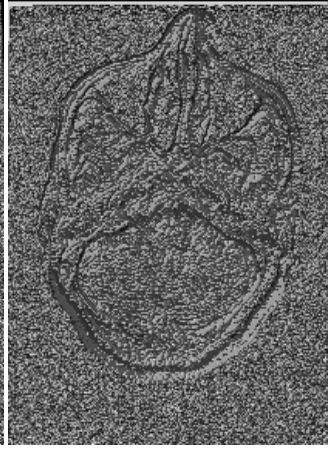

(i)

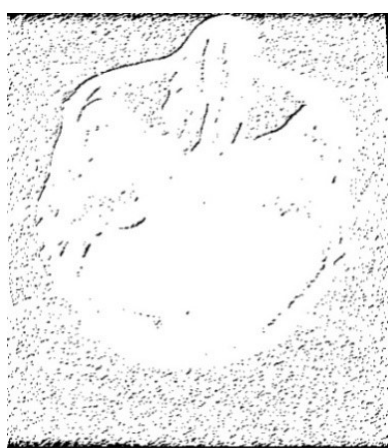

(c)

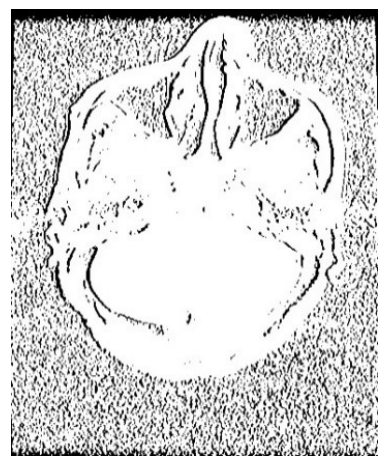

(f)

Figure 6: (a) MR image from data set 1 (b) Image obtained after using directional gradient along 0 degree. (c) Image obtained after using directional gradient along 45 degree. (d) Image obtained after using directional gradient along 90 degree. (e) Image obtained after using directional gradient along 135 degree. (f) Image obtained after using directional gradient along 180 degree. (g) Image obtained when enhanced LMePVEP approach is applied for $j=1$ in forward direction. (h) Image obtained when enhanced LMePVEP approach is applied for $j=2$ in forward direction. (i) Image obtained when enhanced LMePVEP approach is applied for $j=1$ in backward direction. (j) Image obtained when enhanced LMePVEP approach is applied for $j=2$ in backward direction.

Varun Srivastava and Ravindra Purwar

An extension of local mesh peak valley edge based feature descriptor for image retrieval in bio-medical images
ADCAIJ: Advances in Distributed Computing and Artifical Intelligence Journal Regular Issue, Vol. 7 N. 1 (2018), 77-89 elSSN: 2255-2863 - http://adcaij.usal.es Ediciones Universidad de Salamanca - CC BY NC DC 
The proposed algorithm was compared with conventional LMePVEP and DLTerQEP approaches using three previously mentioned datasets in terms of average retrieval precision (ARP) and average retrieval rate (ARR) which are defined in eq. (13) and eq. (14) respectively.

$$
\mathrm{ARP}=\sum_{i=0}^{n} \frac{\mathrm{P}(\mathrm{i})}{\mathrm{n}} \quad \text { for } \mathrm{m} \leq 10
$$

where $\mathrm{P}(\mathrm{i})$ represents the precision and is defined as

$$
\mathrm{P}(\mathrm{i})=\frac{\text { number of relevant images retrieved }}{\text { total number of images retrieved }}
$$

Also $\mathrm{n}$ is the number of images in the database and $\mathrm{m}$ is the number of similar images retrieved from the database.

$$
\text { Similarly ARP }=\sum_{i=0}^{n} \frac{\mathrm{R}(\mathrm{i})}{\mathrm{n}} \quad \text { for } \mathrm{m} \geq 10
$$

where R(i) represents Recall value and it is defined as

$$
\mathrm{R}(\mathrm{i})=\quad \text { number of relevant images retrieved }
$$

total number of relevant images in the database

To retrieve the similar images, a threshold value for Euclidean distance is used. Images having Euclidean distance lesser than threshold are considered as similar images and those having Euclidean distance larger than threshold distance are considered as dissimilar images. This threshold value is chosen dynamically for different datasets. Table 3 shows the performance of proposed enhanced LMePVEP based retrieval technique in terms of ARR for different threshold values and it had been found that the result is optimal for a threshold of 0.5 . Therefore this threshold value is taken to evaluate the performance of proposed retrieval method for dataset 1 .

Table 3: Performance in terms of ARR (\%) for enhanced LMePVEP for dataset 1 using different threshold values.

\begin{tabular}{c|c|c|c|c|c}
\hline Threshold Value & .01 & .02 & .09 & $\mathbf{. 5}$ & .7 \\
\hline ARR (\%) & 67.27 & 80 & 96.36 & $\mathbf{9 9}$ & 60 \\
\hline
\end{tabular}

Similarly the optimal threshold values for other dataset for the proposed method as well as for the remaining image retrieval techniques for different datasets have been obtained which are summarized in Table 4.

Table 4: Optimal threshold values for different retrieval techniques.

\begin{tabular}{c|c|c|c|c}
\hline & LBP & LMePVEP & DLTerQEP & Enhanced LMEPVEP \\
\hline Database 1 & 0.5 & 0.5 & 0.6 & 0.5 \\
\hline Database 2 & 0.0001 & 0.005 & 0.004 & 0.0001 \\
\hline Database 3 & 0.009 & 0.009 & 0.009 & 0.009 \\
\hline
\end{tabular}

Varun Srivastava and Ravindra Purwar An extension of local mesh peak valley edge based feature descriptor for image retrieval in bio-medical images
ADCAIJ: Advances in Distributed Computing and Artifical Intelligence Journal Regular Issue, Vol. 7 N. 1 (2018), 77-89 eISSN: 2255-2863 - http://adcaij.usal.es Ediciones Universidad de Salamanca - CC BY NC DC 
In Table 5 various techniques are compared for all three datasets in terms of ARR and ARP using their optimal threshold values as shown in Table 4.

Table 5: Comparison of different algorithms for three data sets.

\begin{tabular}{c|c|c|c|c|c|c|c|c}
\hline & \multicolumn{2}{|c|}{ LBP } & \multicolumn{2}{c|}{ LMePVEP } & \multicolumn{2}{c|}{ DLTerQEP } & \multicolumn{2}{c}{ Enhanced LMePVEP } \\
\hline Dataset No. & ARP (\%) & ARR (\%) & ARP (\%) & ARR (\%) & ARP (\%) & ARR (\%) & ARP (\%) & ARR (\%) \\
\hline 1 & 86.0 & 90.0 & 94.0 & 95.0 & 99.0 & 99.0 & 99.0 & 99.0 \\
\hline 2 & 51.1 & 78.0 & 57.0 & 86.4 & 55.0 & 83.3 & 56.3 & 88.2 \\
\hline 3 & 56.0 & 73.0 & 60.3 & 80.8 & 68.2 & 81.8 & 79.5 & 89.0 \\
\hline $\begin{array}{c}\text { Average } \\
\text { ARP ARR values }\end{array}$ & 64.3 & 80.33 & 70.4 & 87.4 & 74.06 & 88.03 & 78.26 & 92.06 \\
\hline
\end{tabular}

The average time taken by different algorithms on datasets considered is given in Table 6 .

The time taken by DLTerQEP is maximum since its feature vector length is much bigger. The time taken by LMePVEP and proposed extension is nearly same. LBP takes lesser time due to reduced feature length.

Table 6: Average time taken by various algorithms.

\begin{tabular}{c|c|c}
\hline S.No. & Algorithm & Average Time (in seconds) \\
\hline 1. & LBP & 0.465 \\
\hline 2. & LMePVEP & 1.218 \\
\hline 3. & DLTerQEP & 1.594 \\
\hline 4. & Proposed Extension & 1.221 \\
\hline
\end{tabular}

As evident from table 4, the proposed framework achieved better ARP and ARR values as compared to other algorithms in most of the cases for different databases except one. For dataset 2, though ARP value of enhanced LMePVEP was less than conventional LMePVEP method, but in terms of ARR proposed method is better than it. Further it can be seen that average performance of enhanced LMePVEP is better than all other indexing techniques.

\section{Conclusion and future scope}

In this paper, a bio-medical image retrieval technique using extended LMePVEP features and its performance is compared with three other image retrieval methods - LBP, conventional LMePVEP and DLTerQEP in terms of ARR and ARP parameters for three different datasets. It has been observed that the proposed enhancement in LMePVEP method is not only better than conventional LMePVEP method by $7.86 \%$ for ARP and $4.66 \%$ for ARR in average but also it outperforms other two methods. Moreover in future the approach can also be combined with other spatial algorithms for image retrieval. However, as seen from Table 4, the result for database 2 for our proposed extension in case of ARP is lesser than regular LMePVEP which shows further scope of improvement in the given algorithm.

\section{Disclosures}

No conflicts of interest, financial or otherwise, are declared by all the authors of this manuscript.

Varun Srivastava and Ravindra Purwar An extension of local mesh peak valley edge based feature descriptor for image retrieval in bio-medical images
ADCAIJ: Advances in Distributed Computing and Artifical Intelligence Journal Regular Issue, Vol. 7 N. 1 (2018), 77-89 elSSN: 2255-2863 - http://adcaij.usal.es Ediciones Universidad de Salamanca - CC BY NC DC 


\section{Acknowledgement}

A special thanks to Radiology department of Deen Dayal Upadhyay Hospital, New Delhi, India need to be mentioned for providing the data set of their patients.

\section{References}

Akakin, H. C., \& Gurcan, M. N. (2012). Content-based microscopic image retrieval system for multi-image queries. IEEE transactions on information technology in biomedicine, 16(4), 758-769. DOI: 10.1109/ TITB.2012.2185829

Akgül, C. B., Rubin, D. L., Napel, S., Beaulieu, C. F., Greenspan, H., \& Acar, B. (2011). Content-based image retrieval in radiology: current status and future directions. Journal of Digital Imaging, 24(2), 208-222. DOI: 10.1007/s10278-010-9290-9

Antani, S., Long, L. R., \& Thoma, G. R. (2004, September). Content-based image retrieval for large biomedical image archives. In Proceedings of 11th World Congress on Medical Informatics (MEDINFO) (7-11).

Clark K, Vendt B, Smith K, Freymann J, Kirby J, Koppel P, Moore S, Phillips S, Maffitt D, Pringle M, Tarbox L, Prior F. The Cancer Imaging Archive (TCIA): Maintaining and Operating a Public Information Repository, Journal of Digital Imaging, Volume 26, Number 6, December, 2013, pp 1045-1057. DOI: 10.1007/ s10278-013-9622-7.

Deep, G., Kaur, L., \& Gupta, S. (2016). Directional local ternary quantized extrema pattern: A new descriptor for biomedical image indexing and retrieval. Engineering Science andTechnology, an International Journal. DOI:10.1016/j.jestch.2016.05. 006.

Deep-2, G., Kaur, L., \& Gupta, S. (2016). Biomedical Image Indexing and Retrieval Descriptors: A Comparative Study. Procedia Computer Science,85, 954-961. DOI: 10.1016/j.procs.2016.05.287

Dimitrovski, I., Kocev, D., Kitanovski, I., Loskovska, S., \& Džeroski, S. (2015). Improved medical image modality classification using a combination of visual and textual features. Computerized Medical Imaging and Graphics,39, 14-26. DOI: 10.1016/j.compmedimag.2014.06.005

Do, M. N., \& Vetterli, M. (2002). Wavelet-based texture retrieval using generalized Gaussian density and Kullback-Leibler distance. IEEE transactions on image processing, 11(2), 146-158. DOI: 10.1109/83.982822

Elcap Database: http://www.via.cornell.edu/lungdb.html. Date of last visit: 01st January 2017.

Gibbs, P., \& Turnbull, L. W. (2003). Textural analysis of contrast-enhanced MR images of the breast. Magnetic Resonance in Medicine, 50(1), 92-98. DOI: 10.1002/mrm.10496.

Guo, Z., Zhang, L., \& Zhang, D. (2010). Rotation invariant texture classification using LBP variance (LBPV) with global matching. Pattern recognition, 43(3), 706-719. DOI: 10.1016/j.patcog.2009.08.017

Haralick, R. M., \& Shanmugam, K. (1973). Textural features for image classification. IEEE Transactions on systems, man, and cybernetics, (6), 610-621.

Heikkilä, M., Pietikäinen, M., \& Schmid, C. (2009). Description of interest regions with local binary patterns. Pattern recognition, 42(3), 425-436.

Hussain, S. U., Napoléon, T., \& Jurie, F. (2012). Face recognition using local quantized patterns. In British Machive Vision Conference (pp. 11-pages).

Kalpathy-Cramer, J., de Herrera, A. G. S., Demner-Fushman, D., Antani, S., Bedrick, S., \& Müller, H. (2015). Evaluating performance of biomedical image retrieval system- An overview of the medical image retrieval task at ImageCLEF 2004- 2013. Computerized Medical Imaging and Graphics, 39, 55-61. DOI: 10.1016/j. compmedimag.2014.03.004.

Kasturi, R., \& Jain, R. (2002). A survey on the use of pattern recognition methods for abstraction, indexing and retrieval of images and video. Pattern recognition, 35(4), 945-965. DOI: 10.1016/S0031-3203(01) 00086-3

Kirk, S., Lee, Y., Roche, C., Bonaccio, E., Filippini, J., \& Jarosz, R. (2016). Radiology Data from The Cancer Genome Atlas Thyroid Cancer [TCGA-THCA] collection.The Cancer Imaging Archive. http://doi. org/10.7937/K9/ TCIA.2016. 9ZFRVF1B

Varun Srivastava and Ravindra Purwar

An extension of local mesh peak valley edge based feature

descriptor for image retrieval in bio-medical images
ADCAIJ: Advances in Distributed Computing and Artifical Intelligence Journal Regular Issue, Vol. 7 N. 1 (2018), 77-89 eISSN: 2255-2863 - http://adcaij.usal.es Ediciones Universidad de Salamanca - CC BY NC DC 
Manjunath, K. N., Renuka, A., \& Niranjan, U. C. (2007). Linear models of cumulative distribution function for content-based medical image retrieval. Journal of medical systems, 31(6), 433-443. DOI: 10.1007/ s10916-007-9075-y.

Müller, H., Kalpathy-Cramer, J., Eggel, I., Bedrick, S., Radhouani, S., Bakke, B \& Hersh, W. (2009, September). Overview of the CLEF 2009 medical image retrieval track.In Workshop of the Cross-Language Evaluation Forum for European Languages (pp. 72-84).Springer Berlin Heidelberg. DOI: 10.1007/978-3-642-15751-6_8.

Murala, Subrahmanyam, R. P. Maheshwari, and R. Balasubramanian. "Local tetra patterns: a new feature descriptor for content-based image retrieval." IEEE Transactions on Image Processing 21.5 (2012): 28742886. DOI: 10.1109/TIP.2012.2188809. DOI: 10.1109/TIP.2012.2188809

Murala, S., \& Wu, Q. J. (2014). Local mesh patterns versus local binary patterns: biomedical image indexing and retrieval. IEEE journal of biomedical and health informatics, 18(3), 929-938. DOI: 10.1109/ JBHI.2013.2288522

Murala-2, S., \& Wu, Q. J. (2014). MRI and CT image indexing and retrieval using local mesh peak valley edge patterns. Signal processing: image communication, 29(3), 400-409. DOI: 10.1016/j.image.2013.12.002.

Ojala, T., Pietikäinen, M., \& Harwood, D. (1996). A comparative study of texture measures with classification based on featured distributions. Pattern recognition, 29(1), 51-59. DOI: 10.1016/0031-3203(95)00067-4.

Quddus, A., \& Basir, O. (2012). Semantic image retrieval in magnetic resonance brain volumes. IEEE transactions on information technology in biomedicine, 16(3), 348-355. DOI: 10.1109/TITB.2012.2189439.

Rao, L. K., \& Rao, D. V. (2015). Local quantized extrema patterns for content-based natural and texture image retrieval. Human-centric computing and Information Sciences, 5(1), 1. DOI: 10.1186/s13673-015-0044-z.

Rahman, M. M., Antani, S. K., \& Thoma, G. R. (2011). A learning-based similarity fusion and filtering approach for biomedical image retrieval using SVM classification and relevance feedback. IEEE Transactions on Information Technology in Biomedicine, 15(4), 640-646. DOI: 10.1109/TITB.2011.2151258.

Scott, G., Shyu, C., R., Knowledge-driven multi-dimensional indexing structure for biomedical media database retrieval. IEEE Trans. Inf. Technol. Biomed.2007; 11 (3): pp. 320-331. DOI: 10.1109/TITB.2006.880551

Tan, X., \& Triggs, B. (2010). Enhanced local texture feature sets for face recognition under difficult lighting conditions. IEEE transactions on image processing, 19(6), 1635-1650. DOI: 10.1109/TIP.2010.2042645.

Unay, D., Ekin, A., \& Jasinschi, R. (2008, October). Medical image search and retrieval using local binary patterns and KLT feature points. In 2008 15th IEEE International Conference on Image Processing (pp. 997-1000) IEEE. DOI: 10.1109/ICIP.2008.4711925.

Vipparthi, S. K., \& Nagar, S. K. (2015). Directional local ternary patterns for multimedia image indexing and retrieval. International Journal of Signal and Imaging Systems Engineering, 8(3), 137-145. DOI: 10.1504/ IJSISE.2015.070485

Vipparthi, S. K., Murala, S., Gonde, A. B., \& Wu, Q. J. (2016). Local directional mask maximum edge patterns for image retrieval and face recognition. IET Computer Vision, 10(3), 182-192. DOI:10.1049/ iet-cvi.2015.0035.

Vipparthi-2, S. K., Murala, S., Nagar, S. K., \& Gonde, A. B. (2015). Local Gabor maximum edge position octal patterns for image retrieval. Neurocomputing, 167, 336-345. DOI: 10.1016/j.neucom.2015.04.062

Vogel, J., \& Schiele, B. (2007). Semantic modeling of natural scenes for content-based image retrieval. International Journal of Computer Vision, 72(2), 133-157. DOI: 10.1007/s11263-006-8614-1.

Zhang, L., Zhou, Z., \& Li, H. (2012, September). Binary Gabor pattern: An efficient and robust descriptor for texture classification. In 2012 19th IEEE International Conference on Image Processing (pp. 81-84). IEEE. DOI: 10.1109/ICIP.2012.6466 800

Varun Srivastava and Ravindra Purwar

An extension of local mesh peak valley edge based feature

descriptor for image retrieval in bio-medical images
ADCAIJ: Advances in Distributed Computing and Artifical Intelligence Journal Regular Issue, Vol. 7 N. 1 (2018), 77-89 eISSN: 2255-2863 - http://adcaij.usal.es Ediciones Universidad de Salamanca - CC BY NC DC 